\title{
Kernos
}

Revue internationale et pluridisciplinaire de religion grecque antique

9| 1996

Varia

\section{La cité grecque : modèle historique, entité religieuse et valeur politique}

Discours introductif par E. Moutsopoulos, président du C.I.E.R.G.A.

\section{Evanghelos A. Moutsopoulos}

\section{(2) OpenEdition}

Journals

Édition électronique

URL : http://journals.openedition.org/kernos/1152

DOI : 10.4000/kernos. 1152

ISSN : 2034-7871

Éditeur

Centre international d'étude de la religion grecque antique

\section{Édition imprimée}

Date de publication : 1 janvier 1996

ISSN : 0776-3824

Référence électronique

Evanghelos A. Moutsopoulos, « La cité grecque : modèle historique, entité religieuse et valeur politique », Kernos [En ligne], 9 | 1996, mis en ligne le 21 avril 2011, consulté le 20 avril 2019. URL http://journals.openedition.org/kernos/1152; DOI : 10.4000/kernos.1152 


\section{La cité grecque : modèle historique, entitê religieuse et valeur politique}

discours prononcé par E. MOUTSOPOULOS, Président du C.I.E.R.G.A.

Je suis heureux que l'occasion me soit donnée aujourd'hui encore de m'adresser aux participants d'un des nombreux colloques internationaux, en l'occurrence le cinquième, organisés par le C.I.E.R.G.A., qui se tient pour la deuxième fois à Athènes. Le caractère interdisciplinaire des intérêts du C.I.E.R.G.A. et de la thématique de ses colloques attirent toujours en Grèce, en Belgique, mais aussi ailleurs, des chercheurs de qualité provenant de domaines voisins et intéressés par le facteur religieux dans la Grèce antique, intensément présent au niveau de la vie privée et sociale des anciens Grecs au cours de leur histoire. En se référant particulièrement à la vie sociale, on ne peut dissocier la dimension politique de la dimension religieuse. Si le rôle de la religion est un rôle conjonctif des communautés, il rencontre sur ce plan le rôle de la polis telle qu'elle surgira à la suite de processus conduisant à la constitution des structures sociales de l'époque.

Par ailleurs, si la réalité religieuse en Grèce présenta d'emblée tous les caractères de la religiosité primitive que l'on décèle même de nos jours dans les sociétés de type archaïque, et dont elle domine la mentalité, elle ne cessa nullement pour autant, au cours d'étapes historiques ultérieures, quand les cités s'étaient déjà consolidées, voire quand leur importance tendit à s'atténuer au profit d'entités étatiques plus amples, de présenter des propriétés témoignant de sa provenance magique et de son articulation pour ainsi dire syncrétique à l'intérieur de laquelle des consentements contradictoires se reflétèrent. Création à partir de processus qui se recouvrent de manière simultanée ou successive, la religion grecque antique ne s'imposa jamais sous forme de dogme amovible; c'est pourquoi elle ne s'opposa que rarement à des tendances politiques, alors qu'elle s'offrit facilement à soutenir des institutions officielles.

C'est à partir des débris des états décrits par Homère, et dans un espace limité par l'existence de peuples barbares, qu'après l'« invasion des Doriens », qui marqua les débuts de l'ère historique nouvelle, la cité grecque prit naissance. En Grèce même, mais aussi parsemées dans l'ensemble du bassin méditerranéen sous forme de colonies, une série de formations réelles, bientôt autonomes, dotées de constitutions et de législations qui en définissaient la structure et le fonctionnement, émergèrent un peu partout. 
Certaines de ces cités ont pu prolonger la vie d'établissements mycéniens préexistants; d'autres s'affirmèrent au gré de nouvelles poussées économiques. Or toutes, à quelques exceptions près, présentaient des traits communs dont le plus important fut qu'elles étaient des associations d'hommes libres, liés par la naissance, les croyances, voire les intérêts, autant que par la conscience d'appartenir, en dépit des particularismes inévitables, en même temps que les membres des autres cités grecques, à une nation et à une culture communes dont témoignaient des institutions telles que les Apatouries attiques, relayés et amplifiés par les amphictyonies.

Toutefois, le propre de la cité grecque fut d'avoir permis à ses membres de se former librement une opinion sur chaque problème de la communauté, et de pouvoir l'exprimer, tout en essayant de persuader de son bien-fondé tous les autres citoyens, et d'en obtenir l'assentiment pour appliquer, d'un commun accord, la solution à ce problème, qui semblerait la plus appropriée compte tenu des circonstances. On décèle dans cette pratique la manifestation la plus concrète de la démocratie directe naissante, notamment celle qui se vit pratiquée à l'agora, à la fois marché et place publique : identification concrétisée dans le vocabulaire qui fit du discours prononcé sur le marché le discours politique par excellence, et qui devint bientôt le pendant du naos.

Les institutions religieuses, militaires et surtout civiles furent d'assez bonne heure complétées par des institutions juridiques et par la formation de corps législatifs régissant les relations entre citoyens, et offrant des cadres de solution aux divers litiges et contentieux. Ce sont ces institutions qui ont défini les conditions ayant favorisé l'éclosion et la floraison des arts et des lettres, puis de la pensée philosophique elle-même. Le passage du muthos au logos supposait en effet l'existence de ces citoyens, de ces hommes libres dont chacun se considérait, tout en considérant chacun des autres, non point comme une personnalité accomplie, c'est-à-dire comme une conscience autonome, à l'image de la cité même, mais bien comme une personne : comme un être et comme une valeur à la fois.

Dans presque tous les cas, ne serait-ce que dans des conditions différentes, maints rites et manifestations dépendant de considérations et de pratiques religieuses furent adoptés par les cités et intégrés dans leur fonctionnement politique et social. Peu nombreux, bien que toujours importants pour la vie culturelle, furent les rites orgiastiques ${ }^{1}$ que les cités n'admirent pas officiellement, sans toutefois manquer de les tolérer pour autant, ne serait-ce qu'en les marginalisant au préalable. Le propre de la religion grecque fut qu'elle ne se fixa jamais quelque dogme formel et rigoureux; d'où sa flexibilité externe, d'où aussi la facilité avec laquelle les mythes religieux se prêtèrent à de nombreuses

1 Cf. E. MOUTSOPOULOS, Prévenir ou guérir? Musique et états orgiastiques chez Platon, in Kernos, 5 (1992), p. 141-151. 
transstructurations, surtout littéraires ${ }^{2}$, mais également plastiques. Par contre, les rites eux-mêmes, strictement respectés en raison de leur nature, demeurèrent tant bien que mal associés à des traditions souvent locales, plus rarement panhelléniques, dont les cités, isolées ou fédérées de quelque manière que ce fût, furent amenées à tenir compte.

C'est à travers la personne de son citoyen que la cité se profile en tant que groupement de consciences indépendantes librement associées en vertu d'une législation aussi stricte que justement appliquée. L'idée de justice ne devint ainsi que la projection de la nécessité de permettre à chaque citoyen de jouir des avantages qui lui étaient offerts par la cité dans les mêmes conditions exactement que tout autre de ses concitoyens, sans distinctions ni privilèges, mais sans contrainte non plus, en toute liberté et en tout respect des prérogatives que la loi lui reconnaissait. On distingue déjà ici les fondements mêmes de la démocratie moderne qui, pour des raisons que l'on ne connaît que trop, ne saura jamais égaler son modèle grec qui se situe en un moment historique précis et unique. Ce moment en fait précisément, du point de vue qualitatif, un modèle supratemporel.

Enfin, et j'y vois l'aspect le plus saillant de la question, sur lequel je désire terminer cette série de réflexions, c'est à l'intérieur de la cité grecque, telle que je viens d'en brosser l'image, sans doute quelque peu schématique, mais non point caricaturale pour autant, à l'intérieur de cette polis que naquit l'art politique. Ce fut, en effet, en tant que génératrice de la notion même de politique, art suprême s'il en fut selon Platon, que la cité grecque s'est affirmée comme valeur politique, autrement dit comme un univers réalisé sans cesse:

En matière de religion, le citoyen grec participe des deux mentalités répertoriées jusqu'ici : de la mentalité scellée par le logos, certes, et que l'aristotélisme représente le mieux jusqu'à nos jours, dominée par le principe de noncontradiction; mais aussi, et en même temps, de la mentalité dite archaïque, régie, elle, par le mythe ${ }^{3}$ et par sa répulsion ferme pour le principe en question $^{4}$. Dans ce cadre, qui n'est pas encore celui du syncrétisme, les pratiques magiques, même collectives, qui survivent en tant qu'incorporées dans des pratiques religieuses, ont littéralement droit de cité tant qu'elles ne se heurtent pas aux dispositions légales relatives à la vie des sociétés. Il en résulte une étonnante plasticité au niveau de leur intégration tacite dans le cérémonial officiel.

2 Gf. ID., La transstructuration du mytbe dans la tragédie, in Du banal au merveilleux. Mélanges L. Jerphagnon, E.N.S. Fontenay, Saint-Cloud, 1989 (Les Cabiers de Fontenay, 55-57), p. 179-188, et Polésis et Tecbnè. Idées pour une philosophie de l'art, 3 : Évocations et résurgences, Montréal, Montmorency, 1994, p. 15-25.

3 Cf. ID., Pbilosophes de l'Égée, Athènes, Fondation de l'Égée, 1991, p. 16-39.

4 Cf. par exemple déjà L. LÉVY-BRUHL, La mentalité primitive, Paris, Alcan, 1922; J. CAzEneuve, La mentalité arcbaique, Paris, A. Colin, 1961, p. 16-23, 73-89. 
Nous parlons de nos jours trop exclusivement d'intégration d'institutions, ou, plus généralement, de réalités à intégrer dans des entités et des ensembles toujours plus amples et plus vastes; nous oublions toutefois de nous référer au modèle exemplaire de la polis, pour y puiser des idées utiles à l'amélioration du niveau qualitatif de la vie des communautés restreintes dont le bien-être ne peut que s'avérer salutaire à l'établissement des structures devant, dans un avenir assez proche, régir la vie de l'humanité à l'échelle planétaire : dialectique dérivant d'un postulat, voire d'un principe d'humanisation des grands ensembles politiques aussi bien que de leurs cellules constitutives.

J'ajouterai que la leçon essentielle à tirer de l'exemple proposé par la cité grecque concerne le statut à la fois existentiel, juridique et politique de ses citoyens : ils ne sont nullement des individus inféodés à une minorité gouvernante; ils sont des personnes épanouies formant une élite universelle. C'est une cité de cette qualité, appartenant à tous et à chacun, où les sciences et les arts trouveraient un terrain propice à leur développement idéal, et où les valeurs communes à toutes les consciences pourraient s'actualiser, que l'on souhaiterait voir renaître sur le sol hellène; une cité exemplaire dont chacun se réclamerait et dont la présence dans le monde contemporain offrirait le type même d'un gouvernement fraternel. Ce n'est point d'une utopie de plus dont il s'agit; la terre, bien réelle, est toujours là, qui attend sa mise en valeur et en appelle à l'esprit des hommes.

Pour toutes ces raisons, la poursuite de la recherche et de l'examen de toutes les données religieuses qui s'inscrivent dans le cadre de la cité grecque archaïque et classique acquiert un intérêt incontestable et peut conduire à des constatations et interprétations nouvelles.

En ma qualité de président du C.I.E.R.G.A., j'ai le privilège de vous souhaiter la bienvenue à Athènes, ainsi qu'un succès éclatant de vos travaux, dans le cadre de ce Colloque. Il est de mon devoir de féliciter, une fois de plus, le Professeur Ioannis Loucas, infatigable promoteur des activités du C.I.E.R.G.A. et organisateur de plusieurs de ses colloques, mais aussi Mme Éveline DurieLoucas, pour leur efficacité qui a permis que ce cinquième colloque du C.I.E.R.G.A. devienne, à son tour, une réalité. Bon séjour à Athènes. Je vous remercie. 